\title{
Proximate composition and energetic value of demersal and pelagic prey species from the SW Atlantic Ocean
}

\author{
E. B. Eder ${ }^{1}$, M. N. Lewis ${ }^{2, *}$ \\ ${ }^{1}$ Universidad Nacional de la Patagonia, San Juan Bosco, Boulevard Brown 5000, (9120) Puerto Madryn, Chubut, Argentina \\ ${ }^{2}$ Centro Nacional Patagónico -CONICET, Boulevard Brown 3500, (9120) Puerto Madryn, Chubut, Argentina
}

\begin{abstract}
This study describes the proximate composition (moisture, lipid, protein and ash content) and energetic value of 39 species ( 27 teleosts, 3 sharks, 7 rays and 2 squids) that are known as key prey or potential prey of top predators on the Patagonian continental shelf. Specimens were collected in the SW Atlantic Ocean in the area 39 to $51^{\circ} \mathrm{S}, 55$ to $65^{\circ} \mathrm{W}$. Fifty-two catches were performed at a depth range of 73 to $370 \mathrm{~m}: 27$ catches in autumn and 25 in summer. A multivariate correspondence analysis showed an association between species identity and certain biochemical variables (main dimension $=83 \%$ of variance explained). Sharks and some demersal-pelagic teleosts were associated with lipids (9 to $18 \%$ wet mass). Teleosts constituted a higher source of protein $(9-28 \%$ wet mass) than elasmobranches. Rays were nutritionally poor, with the lowest lipid contents (2 to $10 \%$ ) and energy values ( 3 to $7 \mathrm{~kJ} \mathrm{~g}^{-1}$ wet mass), in agreement with the general biochemical characteristics of benthic fishes. Demersal-benthic and demersal-pelagic species did not change in their nutritional values with increasing size. However, demersal-benthic species increased seasonally in protein content from $11-16 \%$ in summer to $10-19 \%$ in autumn. The energetic value of teleosts from the SW Atlantic Ocean was not different from those of the Pacific or Indian Southern Ocean, but was higher than the energetic value of teleosts from the Northern Hemisphere. This study contributes to the understanding of the biochemical composition and energetic value of prey species over the seasons and their relevance as potential prey for top predators.
\end{abstract}

KEY WORDS: Nutritional quality · Energetic value · Teleosts · Elasmobranches · Cephalopods · Southwestern Atlantic Ocean

\section{INTRODUCTION}

The Patagonian shelf supports a rich and diverse community of resident top predators as well as seasonal migrants that either winter in the region or that travel to tropical and Northern Hemisphere wintering grounds (Croxall \& Wood 2002). The Argentinean continental shelf system is strongly affected by different types of water masses that promote the recycling of nutrients, accounting for its high productivity (Podesta et al. 1991, Carreto et al. 1995). Several studies of marine mammals and seabirds highlighted the extraordinary importance of this system in sustaining populations breeding at the Patagonian coast, Malvinas/Falkland
Islands and South Georgia Island (Croxall \& Wood 2002, Quintana \& Dell'Arciprete 2002, Campagna \& Croxall 2003). Regions visited by top predators during their foraging phase are assumed to be characterised by higher productivity (Bradshaw et al. 2004), but the energy content of potential food resources is currently unknown.

The nutritional quality of marine organisms is often estimated by the lean mass of commercial species, while nutritional information about whole specimens of commercial and non-commercial species is generally scarce. Therefore, there is a lack of knowledge about the energetic values of potential prey, which are needed, for example, to develop models of energy 
transfer between trophic levels. This study provides the relative dietary values of whole teleost, elasmobranch and cephalopod specimens from the SW Atlantic Ocean for the incorporation into trophic ecological studies, such as the foraging behaviour of predators, the assessment of energy transfer between trophic levels (Croxall \& Prince 1982, Tierney et al. 2002) and the shifting energetic values of the spectrum of prey species.

\section{MATERIALS AND METHODS}

The sampling area was located in the area 39 to $51^{\circ} \mathrm{S}, 55$ to $65^{\circ} \mathrm{W}$ (Fig. 1). Fish and squid samples from the SW Atlantic Ocean were collected in autumn (May-June 2001) and summer (January-February 2002) by commercial fishing vessels operating with bottom trawls. Non-parametric Mann-Whitney $U$ rank tests were performed to test for a difference in the depth of hauls between the 2 seasons (Siegel \& Castellan 1995, Conover 1999). Collected material was frozen on board and later analysed in the laboratory. Thirty-nine species $(27$ teleosts, 3 sharks, 7 rays and 2 squids) were identified, weighed and measured. When smaller and larger specimens were observed, samples were sorted according to size, and size-classes were identified as juvenile or adults if appropriate literature was available (Brunetti et al. 1998, Bezzi et al. 2000, Cosseau \& Perrota 2000). Besides, we assigned each species to an ecological

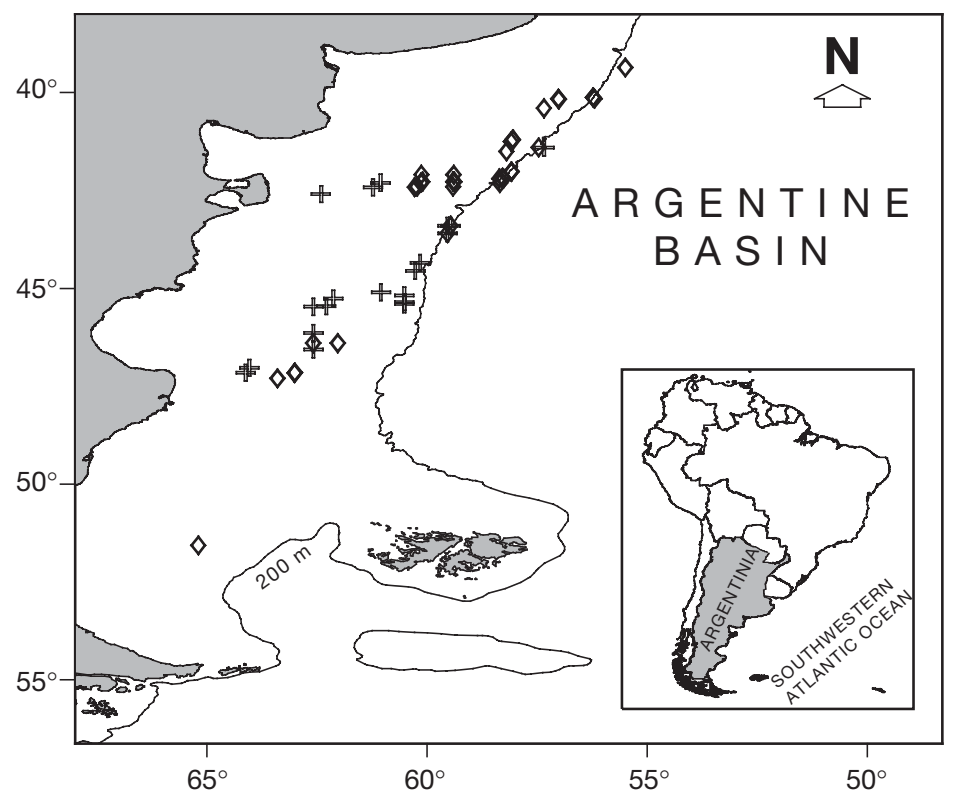

Fig. 1. Locations of catches of teleosts, elasmobranches and cephalopods during autumn $2001(\diamond)$ and summer $2002($ ) from vessels operating with bottom trawls group (pelagic, demersal, demersal-pelagic, demersal-benthic, benthic) according to their habitat and vertical distribution (Brunetti et al. 1998, Bezzi et al. 2000, Cosseau \& Perrota 2000).

Proximate composition and energetic value were determined for 76 samples (44 from autumn and 32 from summer). Samples were dried at $100^{\circ} \mathrm{C}$ until a constant weight was reached. Moisture content was calculated by the difference between wet and dry mass, and then expressed as a percentage of the original mass (Horwitz 1960). Total lipids were extracted with a Soxhlet equipment, using an ether-ethylic solvent. Protein content was determined by the Lowry method (Lowry et al. 1951). Ash content was measured using a muffle furnace at $550^{\circ} \mathrm{C}$ (AKE 1337 $1100^{\circ} \mathrm{C}$ ). Since carbohydrate content is generally low in fish and its contribution to the energetic value is practically zero (Márquez et al. 1996, Payne et al. 1999, Anthony et al. 2000), this component was not measured. The energetic value was determined indirectly - using Rubner's coefficients for aquatic organisms: $9.5 \mathrm{kcal} \mathrm{g}^{-1}$ for lipids, $5.65 \mathrm{kcal} \mathrm{g}^{-1}$ for proteins (Winberg 1971) - and expressed in $\mathrm{kJ} \mathrm{g}^{-1}$ wet mass. All presented nutritional values are means of triplicate determinations (average standard deviation was less than $3 \%$ ).

A multivariate analysis (correspondence analysis) was performed to investigate the association between species and nutritional variables (Digby \& Kempton 1987, Ludwig \& Reynolds 1988, Hair et al. 1999) using a statistical software package (STATISTICA; StatSoft 2001). Non-parametric KruskalWallis and Mann-Whitney $U$ rank tests were performed to test for seasonal differences, differences between adults and juveniles, and differences between ecological or taxonomic groups (Siegel \& Castellan 1995, Conover 1999). In the case when more than 2 groups were compared, we perfomed post-hoc tests in order to determine in more detail how groups differed, using a pairwise comparison method that calculates a statistic $(T)$ based on the differences of ranks of pairs and a critical value $\left(\mathrm{CV}_{i}\right.$ Conover 1999). If $T>C V$, pairs are assumed to be different (Conover 1999).

\section{RESULTS}

Fifty-two catches were performed at a depth range of 73 to $370 \mathrm{~m}$ (Fig. 2). Twenty-seven of these catches were done in autumn (82 to $370 \mathrm{~m}$ ) and 25 in summer (73 to $119 \mathrm{~m}$ ). There was no significant difference in the depth of catches between seasons (Mann-Whitney $U$-test, 
$\left.U_{27,25}=265.5, \mathrm{p}>0.05\right)$. Of all observed species ( $\mathrm{n}=39$ ), $51 \%$ was caught in both seasons $(\mathrm{n}=20)$, $31 \%$ only in autumn $(\mathrm{n}=12)$, and $18 \%$ only in summer $(\mathrm{n}=7)$.

Wet mass ranged from 17 to $4475 \mathrm{~g}$ for teleosts, from 175 to $2425 \mathrm{~g}$ for sharks, from 260 to 3550 $\mathrm{g}$ for rays and from 72 to $1250 \mathrm{~g}$ for squids (Table 1 ). Total length was 14 to $99 \mathrm{~cm}$ for teleosts, 48 to $84 \mathrm{~cm}$ for sharks and 34 to $65 \mathrm{~cm}$ for rays (Table 1). Mantle length of squids ranged from 17 to $42 \mathrm{~cm}$ (Table 1).

Moisture content ranged from 60.5 to $82.5 \%$ and showed an inverse relation with lipid content $(\mathrm{n}=39$, Spearman $\mathrm{R}=-0.8, \mathrm{p}<0.01)$. Lipid and protein content varied among species (Table 2). Ash content was more constant; mean values were $3.1,2.6$ and $2 \%$ for teleosts, elasmobranches and squids respectively (Table 2 ).

The correspondence analysis showed an association of species with 3 biochemical variables. Two main groups associated with lipid (1) or protein (2), and 1 tiny group of teleosts (3) represented by Prionotus nudigula and Cottunculus granulosus stands out mostly due to the contribution of ashes (main dimension $=83 \%$ of variance explained; Fig. 3). The group of species closely associated with lipid is represented by Dissostichus eleginoides, Stromateus brasiliensis, Parona signata, Seriolella punctata, Bathyraja brachyurops, Shroederichthys bivius, Squalus acanthias, and Macruronus magellanicus. The group of species more associated with protein included teleost and ray species, the elasmobranch Callorhynchus callorhynchus and the squids Illex argentinus and Moroteuthis ingens. According to the results, lipid content was higher in the group of species associated with lipid (KruskalWallis test $H_{2,39}=19.18, \mathrm{p}<0.01$; Group 1 vs. Group 2: $T=27.11$, $\mathrm{CV}=13.06$; Group 1 vs. Group 3: $T=17.71, \mathrm{CV}=6.40)$, but there

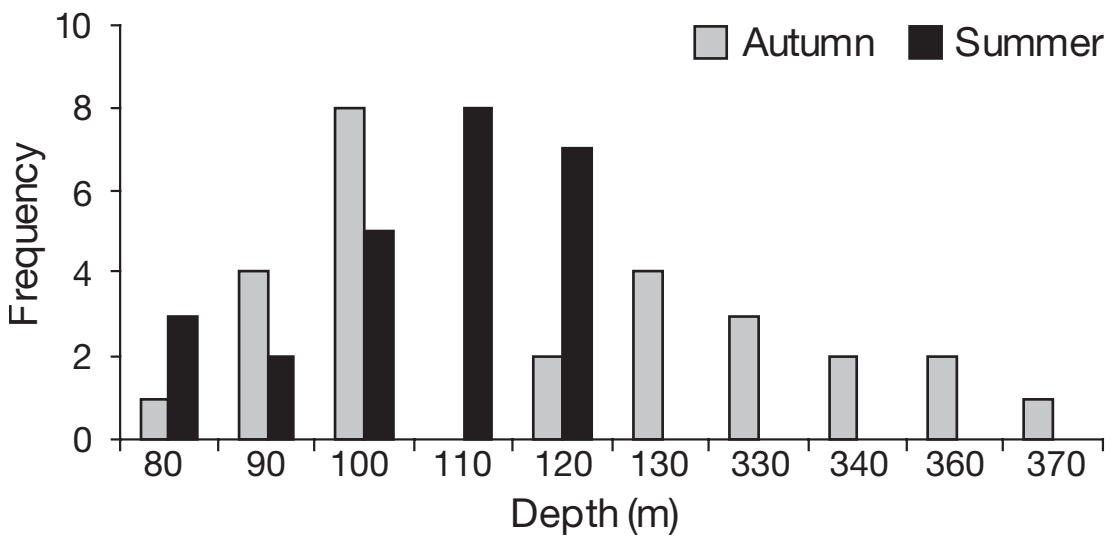

Fig. 2. Distribution of depths of geo-referenced hauls in autumn and summer in the SW Atlantic Ocean

Table 1. Mean wet mass $(\mathrm{g})$ and length $(\mathrm{cm})$ of species from the SW Atlantic Ocean. The ecological group for each species is shown in parentheses $(\mathrm{D}=$ demersal, $\mathrm{DP}=$ demersal-pelagic, $\mathrm{DB}=$ demersal-benthic, $\mathrm{P}=$ pelagic, $\mathrm{B}=$ benthic), $\mathrm{n}=$ number of specimens measured; for species with $\mathrm{n}<3$, wet mass and length are presented in the range column

\begin{tabular}{|c|c|c|c|c|c|}
\hline Species & $\mathrm{n}$ & $\begin{array}{l}\text { Wet mass } \\
\quad(\mathrm{g})\end{array}$ & $\begin{array}{l}\text { Mean } \\
\text { (range) }\end{array}$ & $\begin{array}{l}\text { Length } \\
(\mathrm{cm})\end{array}$ & $\begin{array}{l}\text { Mean } \\
\text { (range) }\end{array}$ \\
\hline \multicolumn{6}{|l|}{ ELASMOBRANCHES } \\
\hline $\begin{array}{l}\text { Squalidae } \\
\text { Squalus acanthias (DB) }\end{array}$ & 22 & 893.2 & $(175-2425)$ & 60.4 & $(47.5-75)$ \\
\hline $\begin{array}{l}\text { Scyliorhinidae } \\
\text { Schroederichthys bivius (DB) }\end{array}$ & 10 & 825.7 & $(447.7-1122.4)$ & 67.2 & $(57.5-75.5)$ \\
\hline $\begin{array}{l}\text { Torpedinidae } \\
\text { Discopyge tschudii (B) }\end{array}$ & 1 & & 795 & & 41.2 \\
\hline $\begin{array}{l}\text { Rajidae } \\
\text { Dipturus chilensis (B) } \\
\text { Psammobatis scobina (B) } \\
\text { Psammobatis normani (B) } \\
\text { Bathyraja brachyurops (B) } \\
\text { Bathyraja macloviana (B) } \\
\text { Bathyraja scaphiops (B) }\end{array}$ & $\begin{array}{l}8 \\
5 \\
8 \\
2 \\
5 \\
3\end{array}$ & $\begin{array}{c}630 \\
874 \\
627.5 \\
\\
595 \\
466.7\end{array}$ & $\begin{array}{c}(320-950) \\
(260-2000) \\
(387.5-872.1) \\
(2900-3550) \\
(300-800) \\
(350-600)\end{array}$ & $\begin{array}{c}50.4 \\
47 \\
45.7 \\
\\
42.8 \\
44.3\end{array}$ & $\begin{array}{c}(41-58.5) \\
(33.5-64.5) \\
(39.5-51.2) \\
(51.5-54) \\
(34.5-48) \\
(40-50)\end{array}$ \\
\hline $\begin{array}{l}\text { Callorhynchidae } \\
\text { Callorhynchus callorhynchus (B) }\end{array}$ & 1 & & 1700 & & 84 \\
\hline TELEOSTS & & & & & \\
\hline $\begin{array}{l}\text { Congridae } \\
\text { Bassanago albescens (DB) }\end{array}$ & 22 & 357.9 & $(212-625)$ & 63.6 & $(56-71)$ \\
\hline $\begin{array}{l}\text { Moridae } \\
\text { Salilota australis (DB) } \\
\text { Austrophycis marginata (DP) }\end{array}$ & $\begin{array}{l}5 \\
3\end{array}$ & $\begin{array}{c}1381.9 \\
38.9\end{array}$ & $\begin{array}{l}(295-4475) \\
(17-62)\end{array}$ & $\begin{array}{l}54.4 \\
17.8\end{array}$ & $\begin{array}{l}(32.4-74) \\
(15-21)\end{array}$ \\
\hline $\begin{array}{l}\text { Gadidae } \\
\text { Micromesistius australis (DP) }\end{array}$ & 4 & 943.8 & $(110.3-1375)$ & 47.5 & $(27-57)$ \\
\hline $\begin{array}{l}\text { Macruronidae } \\
\text { Macruronus magellanicus (DP) }\end{array}$ & 5 & 1095 & $(300-1725)$ & 71.7 & $(49-88)$ \\
\hline $\begin{array}{l}\text { Merlucciidae } \\
\text { Merluccius australis (DP) } \\
\text { Merluccius hubbsi (DP) }\end{array}$ & $\begin{array}{c}9 \\
20\end{array}$ & $\begin{array}{l}2447.8 \\
536.7\end{array}$ & $\begin{array}{c}(725-3625) \\
(175-1457.3)\end{array}$ & $\begin{array}{c}74.9 \\
42\end{array}$ & $\begin{array}{c}(61.5-86) \\
(33-64)\end{array}$ \\
\hline $\begin{array}{l}\text { Macrouridae } \\
\text { Coelorhynchus fasciatus (DB) }\end{array}$ & 16 & 496.7 & $(187.3-1137)$ & 45.9 & $(37-59)$ \\
\hline $\begin{array}{l}\text { Ophidiidae } \\
\text { Genypterus blacodes (DB) }\end{array}$ & 10 & 1303.3 & $(240-3500)$ & 65.1 & $(42-98.5)$ \\
\hline
\end{tabular}

(Table 1 continued on next page) 
Table 1 (continued)

\begin{tabular}{|c|c|c|c|c|c|}
\hline Species & $\mathrm{n}$ & $\begin{array}{l}\text { Wet mass } \\
(\mathrm{g})\end{array}$ & $\begin{array}{c}\text { Mean } \\
\text { (range) }\end{array}$ & $\begin{array}{l}\text { Length } \\
\text { (cm) }\end{array}$ & $\begin{array}{c}\text { Mean } \\
\text { (range) }\end{array}$ \\
\hline $\begin{array}{l}\text { Scorpaenidae } \\
\text { Sebastes oculatus (D) }\end{array}$ & 8 & 486.6 & $(113.9-806.1)$ & 33.3 & $(24.3-38.5)$ \\
\hline $\begin{array}{l}\text { Triglidae } \\
\text { Prionotus nudigula (DB) }\end{array}$ & 7 & 119.1 & $(113-140)$ & 26 & $(25-28)$ \\
\hline $\begin{array}{l}\text { Congiopodidae } \\
\text { Congiopodus peruvianus (D) }\end{array}$ & 14 & 322.3 & $(50-727)$ & 26.5 & $(18-35)$ \\
\hline $\begin{array}{l}\text { Serranidae } \\
\text { Acanthistius brasilianus (DB) }\end{array}$ & 7 & 603.6 & $(410-1000)$ & 34.4 & $(31-41)$ \\
\hline $\begin{array}{l}\text { Bramidae } \\
\text { Brama brama (DP) }\end{array}$ & 9 & 3471.1 & $(3015-3920)$ & 61.5 & $(57-63)$ \\
\hline $\begin{array}{l}\text { Zoarcidae } \\
\text { Iluocoetes fimbriatus (D) }\end{array}$ & 7 & 687.5 & $(450-925)$ & 45.5 & $(41-53)$ \\
\hline $\begin{array}{l}\text { Bovichtidae } \\
\text { Cottoperca gobio (D) }\end{array}$ & 10 & 795.2 & $(353-2175)$ & 38.7 & $(31-62)$ \\
\hline $\begin{array}{l}\text { Carangidae } \\
\text { Parona signata (DP) }\end{array}$ & 1 & & 860 & & 73.40 \\
\hline $\begin{array}{l}\text { Cheilodactylidae } \\
\text { Nemadactylus bergi (DB) }\end{array}$ & 13 & 150.2 & $(43-400)$ & 21.6 & $(16-33)$ \\
\hline $\begin{array}{l}\text { Nototheniidae } \\
\text { Dissostichus eleginoides (DP) } \\
\text { Patagonotothen ramsayi (DB) }\end{array}$ & $\begin{array}{c}1 \\
23\end{array}$ & 154.1 & $\begin{array}{c}3700 \\
(23.2-387.3)\end{array}$ & 23.4 & $\begin{array}{c}66.5 \\
(14-33)\end{array}$ \\
\hline $\begin{array}{l}\text { Pinguipedidae } \\
\text { Pseudopercis semifasciata (DB) }\end{array}$ & 13 & 349.4 & $(95-900)$ & 30.3 & $(21-43)$ \\
\hline $\begin{array}{l}\text { Scombridae } \\
\text { Scomber japonicus (DP) }\end{array}$ & 4 & 590 & $(380-725)$ & 39.9 & $(35-43)$ \\
\hline $\begin{array}{l}\text { Centrolophidae } \\
\text { Seriolella punctata (DP) }\end{array}$ & 8 & 771.8 & $(200-1281)$ & 37.3 & $(28-44)$ \\
\hline $\begin{array}{l}\text { Stromateidae } \\
\text { Stromateus brasiliensis (DP) }\end{array}$ & 13 & 320.2 & $(191-380)$ & 29.1 & $(25-32)$ \\
\hline $\begin{array}{l}\text { Psychrolutidae } \\
\text { Cottunculus granulosus (D) }\end{array}$ & 1 & & 131.7 & & 19 \\
\hline $\begin{array}{l}\text { Paralychthyidae } \\
\text { Paralichthys patagonicus (B) }\end{array}$ & 1 & & 491.4 & & 37 \\
\hline $\begin{array}{l}\text { Achiropsettidae } \\
\text { Mancopsetta maculata (B) }\end{array}$ & 4 & 384.6 & $(273.7-463.8)$ & 34.5 & $(29.3-39)$ \\
\hline $\begin{array}{l}\text { CEPHALOPODS } \\
\text { Ommastrephidae } \\
\text { Illex argentinus (P) }\end{array}$ & 22 & 316.9 & $(71.9-625)$ & 24.7 & $(16.5-33)$ \\
\hline $\begin{array}{l}\text { Onychoteuthidae } \\
\text { Moroteuthis ingens (P) }\end{array}$ & 1 & & 1250 & & 41.5 \\
\hline
\end{tabular}

$\mathrm{CV}=14.30 ;$ rays vs. sharks: $T=$ $31.81, \mathrm{CV}=20.41$; rays vs. squids: $T=30.10, \mathrm{CV}=22.75)$. Comparing ecological groups (Table 1), benthic species showed the lowest lipid contents $\left(H_{3,66}=12.67, \mathrm{p}<0.05\right.$; benthic vs. demersal-pelagic: $T=23.08$, $\mathrm{CV}=12.44$; benthic vs. demersalbenthic: $T=12.56, \quad C V=11.90$; benthic vs. pelagic: $T=22.05, \mathrm{CV}=$ 18.55), the lowest energetic values $\left(H_{3,66}=15.91, \mathrm{p}<0.01\right.$; benthic vs . demersal-pelagic: $T=26.77, \mathrm{CV}=$ 12.04; benthic vs. demersal-benthic: $T=16.64, \mathrm{CV}=11.52$; benthic vs. pelagic $T=21.56, \mathrm{CV}=17.96$ ) and the highest moisture contents $\left(H_{3,66}=15.43, \mathrm{p}<0.01\right.$; benthic vs. demersal-pelagic: $T=26.17, \mathrm{CV}=$ 12.10; benthic vs. demersal-benthic: $T=18.55, \mathrm{CV}=11.58$; benthic vs. pelagic: $T=21.87, \mathrm{CV}=18.05)$. Demersal-benthic species showed lower lipid values than demersalpelagic species $(T=10.52, \mathrm{CV}=$ 10.26).

Lipid contents of demersal-benthic and demersal-pelagic groups did not differ between adults and juveniles: 5 vs. $4 \%$ in demersal-benthic (Mann-Whitney $U$-test, $U_{9,9}=29.00$, $\mathrm{p}>0.05), 10$ vs. $7 \%$ in demersalpelagic $\left(U_{5,5}=10.00, \mathrm{p}>0.05\right)$. Although formal statistical testing was impossible with only 2 samples, it was striking that Nemadactylus bergi adults showed a lipid content of $22 \%$, while juveniles showed a lipid content of only $5 \%$. Protein content was similar between adults and juveniles: 16 vs. $14 \%$ in demersalbenthic $\left(U_{9,9}=23.00, \mathrm{p}>0.05\right)$ and 13 vs. $14 \%$ in demersal-pelagic $\left(U_{5,5}=12.00, \mathrm{p}>0.05\right)$. Demersal-benthic species showed an increase in protein content in autumn $\left(U_{9,9}=18.00, \mathrm{p}<0.05\right.$; Table 3$)$.

\section{DISCUSSION}

This work reports the nutritional values of several species cited as prey of some marine mammals and seabirds from the Patagonian coast (Table 4) and that are more frequently caught by commercial fishing vessels. Despite the diversity of potential prey and 


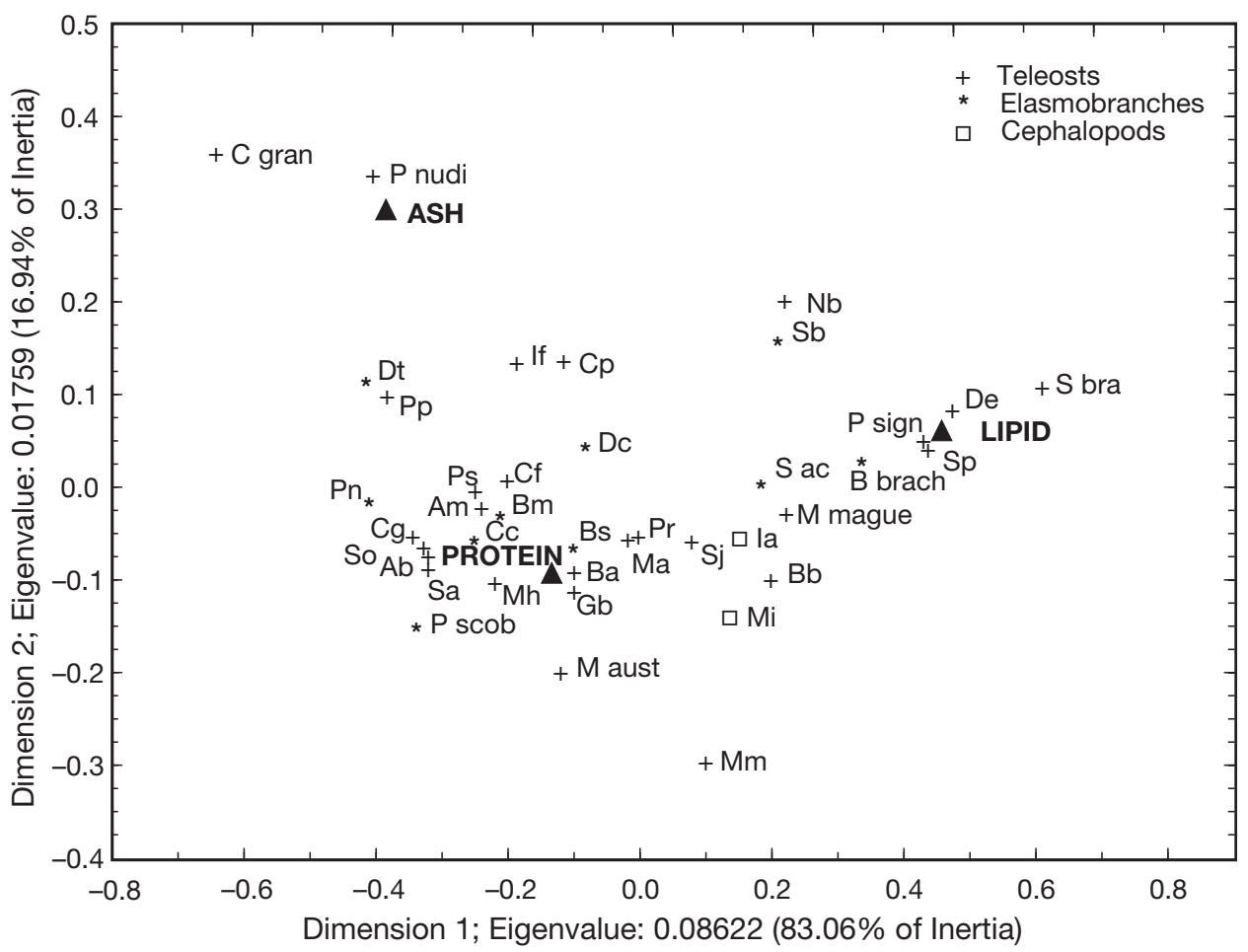

Fig. 3. Results of a multivariate correspondence analysis between some nutritional components and species identity. Samples from summer and autumn, and adults and juveniles, were averaged for each species. Species identity labels: Ab, Acanthisitius brasilianus; Am, Austrophycis marginata; Ba, Bassanago albescens; B brach, Bathyraja brachyurops; Bb, Brama brama; Bm, B. macloviana; Bs, B. scaphiops; Cc, Callorhynchus callorhynchus; Cf, Coelorhynchus fasciatus; C gran, Cottunculus granulosus; Cg, Cottoperca gobio; Cp, Congiopodus peruvianus; Dc, Dipturus chilensis; De, Dissostichus eleginoides; Dt, Discopyge tschuddi; Gb, Genypterus blacodes; Ia, Illex argentinus; If, Iluocoetes fimbriatus; M mague, Macruronus magellanicus; Mm, Mancopsetta maculata; M aust, Merluccius australis; Ma, Micromesistius australis; Mi, Moroteuthis ingens Mh, M. hubbsi; Nb, Nemadactylus bergi; P nudi, Prionotus nudigula; Pn, P. normani; Pp, Paralichthys patagonicus; P scob, Psammobatis scobina; P sign, Parona signata; Pr, Patagonotothen ramsayi; Ps, Pseudopercis semifasciata; S ac, Squalus acanthias; Sa, Salilota australis; Sb, Schroederichthys bivius; S bra, Stromateus brasiliensis; Sj, Scomber japonicus; So, Sebastes oculatus; Sp, Seriolella punctata

the nutritional quality of different species in the SW Atlantic Ocean system, only the Ar-gentine hake Merluccius hubbsi and the Argentine shortfin squid Illex argentinus were described as important contributors to the diet of most predators, with a relative importance index greater than $10 \%$ (Table 4).

Thirty-eight percent of the species analysed here are commercially important. Only the Argentine hake Merluccius hubbsi was a target species for bottom trawler vessels, and the remaining $62 \%$ of the species are by-catch (Bezzi et al. 2000, Cosseau \& Perrotta 2000). Seasonal migration of some species and the seasonal distribution of catches may explain why some species were sampled in only 1 season, e.g. Dissostichus eleginoides and Moroteuthis ingens, which are targeted in autumn with specific fishing gear, or Micromesistius australis, whose summer distribution is
Table 2. Mean proximate composition (\% wet mass) and energetic value ( $\mathrm{kJ} \mathrm{g}^{-1}$ wet mass) of species from the SW Atlantic Ocean

\begin{tabular}{|c|c|c|c|c|c|}
\hline Species & Moisture & Ash & Lipid & Protein & $\begin{array}{l}\text { Energetic } \\
\text { value }\end{array}$ \\
\hline \multicolumn{6}{|l|}{ ELASMOBRANCHES } \\
\hline $\begin{array}{l}\text { Squalidae } \\
\text { Squalus acanthias }\end{array}$ & 72.79 & 2.31 & 8.55 & 12.79 & 6.42 \\
\hline Scyliorhinidae & & & & & \\
\hline Schroederichthys bivius & 72.21 & 3.35 & 9.36 & 11.12 & 6.34 \\
\hline $\begin{array}{l}\text { Torpedinidae } \\
\text { Discopyge tschudii }\end{array}$ & 82.50 & 3.05 & 1.58 & 8.67 & 2.68 \\
\hline Rajidae & & & & & \\
\hline Dipturus chilensis & 78.06 & 2.75 & 4.42 & 10.51 & 4.23 \\
\hline Psammobatis scobina & 77.73 & 2.36 & 2.05 & 12.56 & 3.79 \\
\hline Psammobatis normani & 77.90 & 3.31 & 1.87 & 12.13 & 3.61 \\
\hline Bathyraja brachyurops & 75.41 & 1.92 & 10.00 & 11.66 & 6.72 \\
\hline Bathyraja macloviana & 77.64 & 2.69 & 3.22 & 11.68 & 4.04 \\
\hline Bathyraja scaphiops & 78.39 & 2.65 & 2.97 & 11.79 & 3.97 \\
\hline $\begin{array}{l}\text { Callorhynchidae } \\
\text { Callorhynchus } \\
\text { callorhynchus }\end{array}$ & 77.18 & 2.09 & 3.89 & 10.79 & 4.09 \\
\hline $\begin{array}{l}\text { TELEOSTS } \\
\text { Congridae } \\
\text { Bassanago albescens }\end{array}$ & 75.78 & 258 & 476 & 1414 & 525 \\
\hline
\end{tabular}

Table 2 continued on next page 
Table 2 (continued)

\begin{tabular}{|c|c|c|c|c|c|}
\hline Species & Moisture & Ash & Lipid & Protein & $\begin{array}{l}\text { Energetic } \\
\text { value }\end{array}$ \\
\hline \multicolumn{6}{|l|}{ Moridae } \\
\hline Salilota australis & 77.40 & 3.15 & 2.62 & 14.39 & 4.44 \\
\hline Austrophycis marginata & 73.48 & 3.95 & 4.15 & 16.26 & 4.91 \\
\hline \multicolumn{6}{|l|}{ Gadidae } \\
\hline Micromesistius australis & 73.88 & 2.47 & 5.55 & 13.42 & 5.38 \\
\hline \multicolumn{6}{|l|}{ Macruronidae } \\
\hline Macruronus magellanicus & $s 72.82$ & 2.07 & 8.59 & 13.14 & 6.51 \\
\hline \multicolumn{6}{|l|}{ Merlucciidae } \\
\hline Merluccius australis & 76.08 & 2.03 & 4.48 & 15.66 & 5.48 \\
\hline Merluccius hubbsi & 77.27 & 2.84 & 3.65 & 14.65 & 4.91 \\
\hline \multicolumn{6}{|l|}{ Macrouridae } \\
\hline Coelorhynchus fasciatus & 76.44 & 3.44 & 4.00 & 13.34 & 4.74 \\
\hline \multicolumn{6}{|l|}{ Ophidiidae } \\
\hline Genypterus blacodes & 76.67 & 2.59 & 5.02 & 15.28 & 5.60 \\
\hline \multicolumn{6}{|l|}{ Scorpaenidae } \\
\hline Sebastes oculatus & 73.88 & 3.85 & 2.99 & 16.61 & 5.12 \\
\hline \multicolumn{6}{|l|}{ Triglidae } \\
\hline Prionotus nudigula & 70.29 & 6.94 & 3.62 & 13.36 & 4.63 \\
\hline \multicolumn{6}{|l|}{ Congiopodidae } \\
\hline Congiopodus peruvianus & 74.33 & 4.00 & 5.26 & 12.21 & 5.00 \\
\hline \multicolumn{6}{|l|}{ Serranidae } \\
\hline Acanthistius brasilianus & 76.36 & 2.79 & 2.27 & 12.41 & 3.82 \\
\hline \multicolumn{6}{|l|}{ Bramidae } \\
\hline Brama brama & 67.66 & 2.09 & 10.85 & 18.38 & 8.65 \\
\hline \multicolumn{6}{|l|}{ Zoarcidae } \\
\hline Iluocoetes fimbriatus & 78.15 & 3.00 & 3.22 & 8.82 & 3.36 \\
\hline \multicolumn{6}{|l|}{ Bovichtidae } \\
\hline Cottoperca gobio & 74.78 & 3.36 & 2.49 & 13.93 & 4.28 \\
\hline \multicolumn{6}{|l|}{ Carangidae } \\
\hline Parona signata & 62.43 & 2.49 & 16.24 & 16.23 & 10.27 \\
\hline \multicolumn{6}{|l|}{ Cheilodactylidae } \\
\hline Nemadactylus bergi & 62.83 & 4.98 & 13.25 & 15.13 & 8.83 \\
\hline \multicolumn{6}{|l|}{ Nototheniidae } \\
\hline Dissostichus eleginoides & 61.42 & 2.50 & 17.06 & 15.64 & 10.46 \\
\hline Patagonotothen ramsayi & 73.06 & 3.10 & 7.36 & 17.07 & 6.88 \\
\hline \multicolumn{6}{|l|}{ Pinguipedidae } \\
\hline Pseudopercis semifasciata & a 73.94 & 3.70 & 3.84 & 14.56 & 4.97 \\
\hline \multicolumn{6}{|l|}{ Scombridae } \\
\hline Scomber japonicus & 69.66 & 2.62 & 8.13 & 16.23 & 7.06 \\
\hline \multicolumn{6}{|l|}{ Centrolophidae } \\
\hline Seriolella punctata & 63.96 & 2.15 & 14.78 & 14.83 & 9.36 \\
\hline Stromateidae & & & & & \\
\hline Stromateus brasiliensis & 67.25 & 1.86 & 17.48 & 12.48 & 9.92 \\
\hline Psychrolutidae & & & & & \\
\hline Cottunculus granulosus & 77.76 & 6.08 & 0.97 & 11.01 & 2.99 \\
\hline Paralychthyidae & & & & & \\
\hline Paralichthys patagonicus & 76.17 & 4.05 & 2.27 & 12.11 & 3.77 \\
\hline Achiropsettidae & & & & & \\
\hline Mancopsetta maculata & 60.55 & 1.06 & 11.29 & 27.90 & 11.45 \\
\hline CEPHALOPODS & & & & & \\
\hline Ommastrephidae & & & & & \\
\hline Illex argentinus & 72.52 & 2.05 & 7.90 & 13.88 & 6.42 \\
\hline Onychoteuthidae & & & & & \\
\hline Moroteuthis ingens & 77.24 & 1.34 & 6.54 & 12.81 & 5.64 \\
\hline
\end{tabular}

centred around the Falkland Islands, but whose distribution during autumn-winter is much wider (Cosseau \& Perrotta 2000). Another case is Scomber japonicus, which migrates to coastal waters during summer (Cosseau \& Perrotta 2000), coinciding with the displacement of the catches during summer (see Fig. 1). Besides their temporally variable geographic distribution, these species have the capacity to move vertically between intermediate and superficial water layers, making them potential prey of both deep-water-feeding and surface-water-feeding predators. Many of these species are distributed in close relation to the cold waters of the Malvinas/ Falkland Current, which is also where petrels, albatrosses and elephant seals forage (Campagna et al. 1998, 1999, Nicholls et al. 2002, Quintana \& Dell'Arciprete 2002, Campagna \& Croxall 2003).

One of the 2 main prey species for top predators, Merluccius hubbsi, is one of the most abundant resources in the area, but also one of the most targeted species by fisheries. In fact, it is an over-fished resource that is near to collapse (Bezzi et al. 2000). Hence, it is useful to have data on nutritional values of other species, since it can help us to understand changes in trophic responses of predators as consequence of seasonal changes in food availability. Trites \& Donelly (2003) have shown strong evidence for a relationship between the decline of Steller's sea lions Eumetopias jubatus in the Gulf of Alaska and Aleutian Islands between the late 1970s and 1990s and the reduced availability of suitable prey, which may have been caused by a reduced abundance of several key species of high nutritional quality. In the SW Atlantic Ocean, there is no evidence for declining populations of top predators, perhaps because this system is offering a broader spectrum of prey of high energetic value. Teleost fish species in the SW Atlantic Ocean have higher energetic values than those from the Gulf of Alaska in the Northern Pacific Ocean (Kruskal-Wallis test $H_{2,61}=11.16, \mathrm{p}<0.05 ; T=11.15, \mathrm{VC}=$ 9.46; Table 5). Our energetic values for teleosts were similar to values of Myctophidae species reported in other Southern Ocean studies (Atlantic Ocean: 6.6 to $9.1 \mathrm{~kJ}$ $\mathrm{g}^{-1}$, Clark \& Prince 1980; Indian Ocean: 7.4 
Table 3. Seasonal comparison of mean proximate components ( $\%$ wet mass) and energetic values ( $\mathrm{kJ} \mathrm{g}^{-1}$ wet mass) of demersal-benthic and demersalpelagic species $(\mathrm{n}=$ total number of specimens included in the samples); $\mathrm{ns}=$

\begin{tabular}{|c|c|c|c|c|c|c|c|}
\hline Species & $\mathrm{n}$ & Season & Moisture & Ash & Lipid & Protein & $\begin{array}{c}\text { Energetic } \\
\text { value }\end{array}$ \\
\hline \multicolumn{8}{|c|}{ ELASMOBRANCHES } \\
\hline $\begin{array}{l}\text { Squalus } \\
\text { acanthias }\end{array}$ & $\begin{array}{l}15 \\
7\end{array}$ & $\begin{array}{l}\text { Autumn } \\
\text { Summer }\end{array}$ & $\begin{array}{l}71.47 \\
74.11\end{array}$ & $\begin{array}{l}2.43 \\
2.18\end{array}$ & $\begin{array}{l}9.20 \\
7.92\end{array}$ & $\begin{array}{l}13.51 \\
12.07\end{array}$ & $\begin{array}{l}6.85 \\
5.99\end{array}$ \\
\hline $\begin{array}{l}\text { Schroederichthys } \\
\text { bivius }\end{array}$ & $\begin{array}{l}5 \\
5\end{array}$ & $\begin{array}{l}\text { Autumn } \\
\text { Summer }\end{array}$ & $\begin{array}{l}71.83 \\
72.59\end{array}$ & $\begin{array}{l}3.49 \\
3.20\end{array}$ & $\begin{array}{c}11.62 \\
7.11\end{array}$ & $\begin{array}{l}10.44 \\
11.80\end{array}$ & $\begin{array}{l}7.07 \\
5.61\end{array}$ \\
\hline \multicolumn{8}{|c|}{ TELEOSTS (DEMERSAL-BENTHIC) } \\
\hline $\begin{array}{c}\text { Bassanago } \\
\text { albescens }\end{array}$ & $\begin{array}{c}15 \\
7\end{array}$ & $\begin{array}{l}\text { Autumn } \\
\text { Summer }\end{array}$ & $\begin{array}{l}74.18 \\
77.39\end{array}$ & $\begin{array}{l}2.99 \\
2.16\end{array}$ & $\begin{array}{l}5.23 \\
4.29\end{array}$ & $\begin{array}{l}17.63 \\
10.66\end{array}$ & $\begin{array}{l}6.27 \\
4.22\end{array}$ \\
\hline $\begin{array}{l}\text { Salilota } \\
\text { australis }\end{array}$ & $\begin{array}{l}1 \\
3\end{array}$ & $\begin{array}{l}\text { Autumn } \\
\text { Summer }\end{array}$ & $\begin{array}{l}76.02 \\
79.67\end{array}$ & $\begin{array}{l}3.69 \\
2.83\end{array}$ & $\begin{array}{l}3.88 \\
1.17\end{array}$ & $\begin{array}{l}16.41 \\
11.37\end{array}$ & $\begin{array}{l}5.42 \\
3.15\end{array}$ \\
\hline $\begin{array}{l}\text { Genypterus } \\
\text { blacodes }\end{array}$ & $\begin{array}{l}1 \\
4\end{array}$ & $\begin{array}{l}\text { Autumn } \\
\text { Summer }\end{array}$ & $\begin{array}{l}75.55 \\
78.22\end{array}$ & $\begin{array}{l}2.72 \\
3.15\end{array}$ & $\begin{array}{l}9.99 \\
1.23\end{array}$ & $\begin{array}{l}13.69 \\
12.48\end{array}$ & $\begin{array}{l}7.20 \\
3.44\end{array}$ \\
\hline $\begin{array}{l}\text { Nemadactylus } \\
\text { bergi }\end{array}$ & $\begin{array}{l}1 \\
4\end{array}$ & $\begin{array}{l}\text { Autumn } \\
\text { Summer }\end{array}$ & $\begin{array}{l}60.08 \\
56.32\end{array}$ & $\begin{array}{l}5.52 \\
4.58\end{array}$ & $\begin{array}{l}13.41 \\
21.63\end{array}$ & $\begin{array}{l}17.57 \\
15.94\end{array}$ & $\begin{array}{c}9.47 \\
12.34\end{array}$ \\
\hline $\begin{array}{l}\text { Patagonotothen } \\
\text { ramsayi }\end{array}$ & $\begin{array}{l}11 \\
12\end{array}$ & $\begin{array}{l}\text { Autumn } \\
\text { Summer }\end{array}$ & $\begin{array}{l}71.30 \\
75.14\end{array}$ & $\begin{array}{l}3.33 \\
2.86\end{array}$ & $\begin{array}{c}10.44 \\
4.27\end{array}$ & $\begin{array}{c}18.9 \\
15.24\end{array}$ & $\begin{array}{l}8.46 \\
5.30\end{array}$ \\
\hline Mann-Whitney $U$ & T-test & $U_{9,9}$ & 20.00 & 26.00 & 22.00 & 18.00 & 16.00 \\
\hline Significance & & & ns & ns & $\mathrm{ns}$ & $\mathrm{p}<0.05$ & $\mathrm{p}<0.05$ \\
\hline \multicolumn{8}{|c|}{ TELEOSTS (DEMERSAL-PELAGIC) } \\
\hline $\begin{array}{l}\text { Merluccius } \\
\text { australis }\end{array}$ & $\begin{array}{l}5 \\
2\end{array}$ & $\begin{array}{l}\text { Autumn } \\
\text { Summer }\end{array}$ & $\begin{array}{l}74.74 \\
77.43\end{array}$ & $\begin{array}{l}1.15 \\
2.91\end{array}$ & $\begin{array}{l}6.68 \\
2.28\end{array}$ & $\begin{array}{l}15.02 \\
16.29\end{array}$ & $\begin{array}{l}6.20 \\
4.76\end{array}$ \\
\hline $\begin{array}{l}\text { Merluccius } \\
\text { hubbsi }\end{array}$ & $\begin{array}{c}12 \\
8\end{array}$ & $\begin{array}{l}\text { Autumn } \\
\text { Summer }\end{array}$ & $\begin{array}{c}76.4 \\
78.14\end{array}$ & $\begin{array}{l}2.36 \\
3.26\end{array}$ & $\begin{array}{l}5.36 \\
1.94\end{array}$ & $\begin{array}{l}12.91 \\
16.38\end{array}$ & $\begin{array}{l}5.18 \\
4.64\end{array}$ \\
\hline Brama brama & $\begin{array}{l}7 \\
2\end{array}$ & $\begin{array}{l}\text { Autumn } \\
\text { Summer }\end{array}$ & $\begin{array}{l}67.61 \\
67.71\end{array}$ & $\begin{array}{l}2.06 \\
2.12\end{array}$ & $\begin{array}{l}10.96 \\
10.74\end{array}$ & $\begin{array}{l}17.59 \\
19.16\end{array}$ & $\begin{array}{l}8.51 \\
8.79\end{array}$ \\
\hline $\begin{array}{r}\text { Seriolella } \\
\text { punctata }\end{array}$ & $\begin{array}{l}4 \\
4\end{array}$ & $\begin{array}{l}\text { Autumn } \\
\text { Summer }\end{array}$ & $\begin{array}{l}60.98 \\
66.93\end{array}$ & $\begin{array}{l}2.20 \\
2.11\end{array}$ & $\begin{array}{l}15.13 \\
14.44\end{array}$ & $\begin{array}{l}15.93 \\
13.72\end{array}$ & $\begin{array}{l}9.76 \\
8.96\end{array}$ \\
\hline $\begin{array}{c}\text { Stromateus } \\
\text { brasiliensis }\end{array}$ & $\begin{array}{l}7 \\
3\end{array}$ & $\begin{array}{l}\text { Autumn } \\
\text { Summer }\end{array}$ & $\begin{array}{l}65.56 \\
65.09\end{array}$ & $\begin{array}{l}1.94 \\
1.63\end{array}$ & $\begin{array}{l}17.09 \\
22.28\end{array}$ & $\begin{array}{l}12.13 \\
11.80\end{array}$ & $\begin{array}{c}9.64 \\
11.76\end{array}$ \\
\hline Mann-Whitney $U$ & T-test & $U_{5,5}$ & 9.00 & 5.00 & 10.00 & 12.00 & 11.00 \\
\hline Significance & & & ns & ns & ns & ns & ns \\
\hline
\end{tabular}
not significant

general picture. Benthic species were the lowest in lipid content and consequently had the lowest energetic values and highest moisture contents (although most of them were rays); demersal-pelagic fishes were higher in lipid and energetic values. An exception to this pattern is the benthic flounder Mancopsetta maculata, which showed a high lipid content but a low moisture content. In all cases, we found a general inverse relationship between moisture and lipid content. Some studies have reported this same trend (Clarke et al. 1994, Márquez et al. 1996, Anthony et al. 2000) and have related this to buoyancy adjustments in some cases, but the main reason for the relationship could be the fact that, unlike proteins and carbohydrates, lipids can be stored almost 'dry' (Stryer 1995).

For marine organisms, body composition is variable with respect to geographical location, time of year, size, maturity condition, sex, and feeding regime or ecological habits (Saadettin et al. 1988, Lawson et al. 1998). Some authors have found a reduction of the protein component during the spawning season (Ando \& Hatano 1986). Since spawning is a process of high energy demand and fish generally do not feed during spawning, lipid stores fall as spawning progresses, and the protein component can also be depleted for this purpose. During intensive feeding seasons, the protein

to $13.3 \mathrm{~kJ} \mathrm{~g}^{-1}$, Lea et al. 2002; Pacific Ocean: 5.4 to $10.9 \mathrm{~kJ} \mathrm{~g}^{-1}$, Tierney et al. 2002).

We found that rays had the lowest nutritional value, due to their lower lipid and energy contents. Compared with teleosts and in correspondence with the rest of the elasmobranches, rays also showed a poor protein content. This low nutritional value fits well with the low index of relative importance of elasmobranches in the diet of marine mammals $(0.4 \%$; Koen Alonso et al. 2000). However, and with relevance to biases associated with the techniques commonly implemented in diet reconstruction, shark species had lipid and energetic values that were as high as teleosts and squids.

Typical lean fish species are those that are associated with the sea floor, while pelagic fishes are usually referred to as 'fatty fishes' due to their high lipid content (Murray \& Burt 1969). Our results agree with this levels rise back to normal values. Reinitz et al. (1979) consider that the nutritional composition of food is the most important factor affecting the proximal composition of fish. Unfortunately, knowledge is not complete for all species from the SW Atlantic, and available information is still insufficient to relate autumnal increases in protein and energetic values in demersalbenthic species with spawning or feeding regimes. Another factor affecting proximal components is the gravid condition. Croxall \& Prince (1982) have found in krill that lipid and energetic value was $42 \%$ higher for gravid females than for mature males. The flounder Mancopsetta maculata showed high average lipid, protein and energetic values: this can be explained by the fact that it was the only benthic species whose sample included gravid specimens. In some studies, larger size (or adult) categories tended to have higher lipid 
Table 4. Top predators in the SW Atlantic Ocean and their key prey species

\begin{tabular}{|c|c|c|}
\hline Predators & Prey species & Sources \\
\hline \multicolumn{3}{|l|}{ MARINE MAMMALS } \\
\hline \multicolumn{3}{|l|}{ Cetaceans } \\
\hline Cephalorhynchus & Merluccius hubbsi & Bastida et al. (1988), Crespo et al. (1997) \\
\hline commersonii & Patagonotothen ramsayi & \\
\hline \multirow[t]{3}{*}{ (Commerson's dolphin) } & Stromateus brasiliensis & Crespo et al. (1997) \\
\hline & Illex argentinus & Bastida et al. (1988), Crespo et al. $(1994,1997)$ \\
\hline & Macruronus magellanicus & Bastida et al. (1988) \\
\hline \multirow{3}{*}{$\begin{array}{l}\text { Lagenorhynchus obscurus } \\
\text { (Dusky dolphin) }\end{array}$} & Merluccius hubbsi & Koen Alonso et al. (1998) \\
\hline & Stromateus brasiliensis & \\
\hline & $\begin{array}{l}\text { Notothenia sp. } \\
\text { Illex argentinus }\end{array}$ & Crespo et al. (1994), Koen Alonso et al. (1998) \\
\hline $\begin{array}{l}\text { Delphinus delphis } \\
\text { (Common dolphin) }\end{array}$ & Illex argentinus & Crespo et al. (1994) \\
\hline Globicephala melaena & Illex argentinus & Crespo et al. (1994) \\
\hline (Long-finned pilot whale) & Moroteuthis ingens & \\
\hline Physeter macrocephalus & Illex argentinus & Clarke et al. (1980), Crespo et al. (1994) \\
\hline (Sperm whale) & Moroteuthis ingens & Crespo et al. (1994) \\
\hline \multicolumn{3}{|l|}{ Pinnipeds } \\
\hline \multirow{9}{*}{$\begin{array}{l}\text { Otaria flavescens } \\
\text { (South American sea lion) }\end{array}$} & Merluccius hubbsi & Koen Alonso et al. (2000) \\
\hline & Genypterus blacodes & \\
\hline & Pseudopercis semifasciata & \\
\hline & Stromateus brasiliensis & \\
\hline & Acanthistius brasilianus & \\
\hline & Seriolella punctata & \\
\hline & Iluocoetes fimbriatus & \\
\hline & Nemadactylus bergi & \\
\hline & Illex argentinus & \\
\hline \multicolumn{3}{|l|}{ MARINE BIRDS } \\
\hline $\begin{array}{l}\text { Diomedea exulans } \\
\text { (Wandering albatross) }\end{array}$ & Illex argentinus & Croxall \& Prince (1994) \\
\hline Spheniscus magellanicus & Merluccius hubbsi & Scolaro et al. (1999) \\
\hline (Magellanic penguin) & Illex argentinus & Clarke et al. (1980), Scolaro et al. (1999) \\
\hline \multicolumn{3}{|l|}{ SHARKS } \\
\hline $\begin{array}{l}\text { Galeorhinus galeus } \\
\text { (Tope shark) }\end{array}$ & Stromateus brasiliensis & Cosseau \& Perrotta (2000) \\
\hline
\end{tabular}

and energetic values than smaller size (or juvenile) categories (Croxall \& Prince 1982, Clarke et al. 1994, Anthony et al. 2000). However, other studies have not found such a relationship between size and proximate composition (Payne et al. 1999), and yet another even found the opposite pattern: smaller size-classes showed higher energy contents (Tierney et al. 2002). It seems that we cannot use general rules, and proximal composition depends strongly on the conditions for each species.

The data presented in this study cover a broad spectrum of species and give practical and useful information about the nutritional values of regional species. Since differences in lipid and protein contents have important implications for digestive efficiency, foraging behaviour and dietary preferences of predators (Lawson et al. 1998), further sampling of individual species is needed for the establishment of detailed standards for composition of potential prey.

Feeding habits, foraging movement patterns, and energy and time expenditure are a predator's inte- gration of its requirements and perceptions of the nutritional resources (Robbins 1983). It is also well known that the distribution of marine predators at sea is closely related to food availability and productivity conditions. Bathymetry has been shown to strongly affect the amount and types of fish available for some top predators, and shelves with a higher surface area offer a greater opportunity for demersal foraging (Green \& Burton 1993). These circumstances, plus high productivity conditions such as frontal zones, could help explain why, even while breeding at South Georgia, wandering albatross, giant petrel and white-chinned petrel forage over the Patagonian shelf (Croxall \& Wood 2002). Predators can compensate for variation in prey availability through adjusted selection of prey as a function of their nutritional quality (Krebs \& Davies 1978, Anthony et al. 2000). Knowledge of the nutritional value of food items turns into an important tool when trying to comprehend these adjustments. 
Table 5. Energy content ( $\mathrm{kJ} \mathrm{g}^{-1}$ wet mass) of teleost species of the Northern Pacific Ocean calculated from the lipid and protein values in Payne et al. (1999). Energetic values were calculated using the same coefficients as used in this study (see 'Materials and methods')

\begin{tabular}{|c|c|c|}
\hline \multirow[b]{2}{*}{ Species } & \multicolumn{2}{|c|}{ Northern Ocean } \\
\hline & $\begin{array}{c}\text { Pacific } \\
\text { (Gulf of Alaska) }\end{array}$ & $\begin{array}{c}\text { Pacific } \\
\text { (Bering Sea) }\end{array}$ \\
\hline \multicolumn{3}{|l|}{ Osmeridae } \\
\hline Thaleichthys pacificus & 10.05 & 10.90 \\
\hline Mallotus villosus & 5.30 & 6.50 \\
\hline Hypomesus pretiosus & 7.25 & - \\
\hline Osmerus mordax & - & 6.90 \\
\hline \multicolumn{3}{|l|}{ Ammodytae } \\
\hline Ammodytes hexapterus & 5.23 & 6.12 \\
\hline \multicolumn{3}{|l|}{ Trichodontidae } \\
\hline Trichodon trichodon & 5.11 & 5.30 \\
\hline \multicolumn{3}{|l|}{ Stichaeidae } \\
\hline Lumpenus spp. & 5.40 & - \\
\hline \multicolumn{3}{|l|}{ Gadidae } \\
\hline Theragra chalcogramma & 3.93 & - \\
\hline \multicolumn{3}{|l|}{ Zaproridae } \\
\hline Zaproa silenus & 3.00 & - \\
\hline \multicolumn{3}{|l|}{ Hexagrammidae } \\
\hline monopterygius & 4.94 & - \\
\hline \multicolumn{3}{|l|}{ Clupeidae } \\
\hline Clupea pallasii & 3.43 & - \\
\hline
\end{tabular}

Acknowledgements. We would like to express our gratitude to the Harengus S.A. fishing company for the samples provided, and to Centro Nacional Patagónico (CONICET) and Universidad Nacional de la Patagonia for institutional support. We thank Dr. A. Gosztonyi for his help with fish identification, Lic. N. De Vido for technical assistance in the chemical analyses, and Dr. P. Edelaar for improving the English language of this paper. We also thank the editor and 2 anonymous reviewers for their comments and suggestions.

\section{LITERATURE CITED}

Ando S, Hatano M (1986) Biochemical characteristics of chum salmon muscle during spawning migration. Bull Jpn Soc Sci Fish 52:229-12354

Anthony JA, Roby DD, Turco KR (2000) Lipid content and energy density of forage fishes from the northern Gulf of Alaska. J Exp Mar Biol Ecol 248:53-78

Bastida R, Lichtschein V, Goodall RNP (1988) Food habits of the Cephalorhynchus commersonii off Tierra del Fuego. Rep Int Whaling Comm 9:3-70

Bezzi S, Akselman R, Boschi E (2000) Síntesis del estado actual de las pesquerías marítimas argentinas y de la cuenca del Plata: años 1997-1998, con una actualización de 1999. Publicaciones especiales. Instituto Nacional de Investigación y Desarrollo Pesquero, Mar del Plata

Bradshaw CJA, Hindell MA, Sumner MD, Michael KJ (2004) Loyalty pays: life-history consequences of fidelity to marine foraging regions by elephant seals. Anim Behav 68:1349-1360

Brunetti NE, Ivanovic ML, Beatriz E (1998) Calamares Omastréfidos (Cephalopoda, Omastrephidae). In: Boschi EE (ed) El Mar Argentino y sus recursos pesqueros, Tomo 2. Los moluscos de interés pesquero: cultivos y estrategias reproductivas de bivalvos y equinoideos. Contribución Instituto Nacional de Investigación y Desarrollo Pesquero No. 1044, Mar del Plata

Campagna C, Croxall J (2003) Application of the landscape species approach to the Patagonian Large Marine Ecosystem (The Sea and Sky Project). UICN-WPC, Durban

Campagna C, Quintana F, Le Boeuf BJ, Blackwell S, Crocker DE (1998) Diving behavior and foraging ecology of female southern elephant seals from Patagonia. Aquat Mamm $4: 1-11$

Campagna C, Fedak MA, McConnell BJ (1999) Post-breeding distribution and diving behaviour of adult male southern elephant seals from Patagonia. J Mamm 4:1341-1352

Carreto JI, Lutz VA, Carignan MO, Cucchi Colleoni AD, De Marco SG (1995) Hydrography and chlorophyll-a in the transect from the coast to the shelf break in the Argentinean Sea. Cont Shelf Res 15:315-336

Clarke A, Prince PA (1980) Chemical composition and calorific value of food fed to mollymauk chicks Diomedea melanophris and D. chrysostoma at Bird Island, South Georgia. Ibis 122:488-494

Clarke A, Rodhouse PG, Gore DJ (1994) Biochemical composition in relation to the energetics of growth and sexual maturation in the ommastrephid squid Illex argentinus. Phil Trans R Soc B 344:201-212

Clarke MR, MacLeod N, Castello HP, Pinedo MC (1980) Cephalopod remains from the stomach of a sperm whale stranded at Rio Grande do Sul in Brazil. Mar Biol 59: $235-239$

Conover WJ (1999) Practical nonparametric statistics, 3rd edn. John Wiley and Sons, Chichester

Cosseau MB, Perrota G (2000) Peces marinos de Argentina. biología, distribución y pesca. Instituto Nacional de Investigación y Desarrollo Pesquero, Mar del Plata

Crespo EA, Nepomnaschy P, Koen Alonso M, García NA (1994) Análisis preliminar de la dieta de mamíferos marinos sobre la base de contenidos estomacales y heces. In: Oporto JA (ed) Anales IV Reu Trab Esp Mamíf Acuát América del Sur. Imprenta Universitaria S. A., Universidad Austral de Chile, Valdivia, p 75-88

Crespo EA, Pedraza SN, Dans SL, Koen Alonso M, García NA, Coscarella MA, Schiavini ACM (1997) Direct and indirect effects of the high seas fisheries on the marine mammal populations in the northern and central Patagonian coast. J Northw Atl Fish Sci 22:189-207

Croxall JP, Prince PA (1982) Calorific content of squid (Mollusca: Cephalopoda). Br Antarct Surv Bull 55:27-31

Croxall JP, Prince PA (1994) Dead or alive, night or day: how do albatrosses catch squid? Antarct Sci 6(2):155-162

Croxall JP, Wood AG (2002) The importance of the Patagonian Shelf to top predator species breeding at South Georgia. Aquat Conserv 12:101-118

Digby PGN, Kempton RA (1987) Multivariate analysis of ecological communities. Chapman and Hall, London

Green K, Burton HR (1993) Comparison of the stomach contents of southern elephant seals, Mirounga leonina, at Macquarie and Heard Islands. Mar Mamm Sci 9 (1):10-22

Hair JF Jr, Anderson RE, Tatham RL, Black WC (1999) Análisis multivariante. Prentice Hall Iberia SRL, Madrid

Horwitz W (1960) Official methods of analysis of the Association of Official Agricultural Chemists. AOAC, Washington, DC

Koen Alonso M, Crespo EA, Garcia NA, Pedraza SN, Coscarella MA (1998) Diet of dusky dolphins (Lagenorhynchus obscurus) in waters off Patagonia, Argentina. Fish Bull 96(2):366-374 
Koen Alonso M, Crespo EA, Pedraza SN, Garcia NA, Coscarella MA (2000) Food habits of the South American sea lion (Otaria flavescens) off Patagonia, Argentina. Fish Bull 98(2):250-263

Krebs JR, Davies NB (1978) Behavioural ecology, an evolutionary approach. Sinauer Associates, Sunderland, MA

Lawson JW, Magalhães AM, Miller EH (1998) Important prey species of marine vertebrate predators in the northwest Atlantic: proximate composition and energy density. Mar Ecol Prog Ser 164:13-20

Lea MA, Nichols PD, Wilson G (2002) Fatty acid composition of lipid-rich myctophids and mackerel icefish (Champsocephalus gunnari) - Southern Ocean food-web implications. Polar Biol 25:843-854

Lowry OH, Rosebrough NJ, Farr AL, Randall RJ (1951) Protein measurement with the Folin reagent. J Biol Chem 193:265-275

Ludwig JA, Reynolds JF (1988) Statistical ecology. John Wiley, New York

Márquez MEI, Casaux RJ, Mazzotta AS (1996) Bacalaos antárticos: peces magros de alto contenido proteico. La Industria Carnica Latinoamericana 105:34-39

Murray J, Burt JR (1969) The composition of fish. Torry Advis, Note 38. Tony Research Station, Aberdeen

Nicholls DG, Robertson CJR, Prince PA, Murray MD, Walker KJ, Elliot GP (2002) Foraging niches of three Diomedea albatrosses. Mar Ecol Prog Ser 231:269-277

Payne SA, Johnson BA, Otto RS (1999) Proximate composition of some north-eastern Pacific forage fish species. Fish Oceanogr 8(3):159-177

Podesta GP, Brown OB, Evans RH (1991) The annual cycle of satellite-derived sea surface temperature in the South-

Editorial responsibility: Otto Kinne (Editor-in-Chief), Oldendorf/Luhe, Germany western Atlantic Ocean. Am Meteorol Soc 4:457-467

Quintana F, Dell'Arciprete OP (2002) Foraging grounds of southern giant petrels (Macronectes giganteus) on the Patagonian shelf. Polar Biol 25:159-161

Reinitz GL, Onne LE, Hitzel FN (1979) Variations of body composition and growth among strains of rainbow trout (Salmo gairdneri). Trans Am Fish Soc 108:204-207

Robbins CT (1983) Wildlife feeding and nutrition. A series of monographs and treatises. Academic Press, New York

Saadettin G, Dincer B, Alemdag N, Colak A, Tufekci M (1998) Proximate composition an selected mineral content of commercially important fish species from the Black Sea. J Sci Food Agric 78:337-342

Scolaro JA, Wilson RP, Laurenti S, Kierspel M, Gallelli H, Upto JA (1999) Feeding preferences of the Magellanic penguin over its breeding range in Argentina. Waterbirds 22:104-110

Siegel S, Castellan NJ (1995) Estadística no paramétrica aplicada a las ciencias de la conducta, 4th edn. Editorial Trillas, México DF

Stryer L (1995) Bioquímica. Cuarta edición, Editorial Reverté, Barcelona

StatSoft Inc (2001) Statistica (data analysis software system), version 6 . Microsoft, Tulsa

Tierney M, Hindell M, Goldsworthy S (2002) Energy content of mesopelagic fish from Macquarie Island. Antarct Sci 14(3):225-230

Trites AW, Donnelly CP (2003) The decline of Steller sea lions Eumetopias jubatus in Alaska: a review of the nutritional stress hypothesis. Mammal Rev 33(1):3-28

Winberg GC (1971) Methods for estimation of production of aquatic animals. Academic Press, New York

Submitted: April 5, 2004; Accepted: January 27, 2005

Proofs received from author(s): March 30, 2005 